\title{
Distributed Containment Control for Multiple Autonomous Vehicles With Double-Integrator Dynamics: Algorithms and Experiments
}

\author{
Yongcan Cao, Student Member, IEEE, Daniel Stuart, Wei Ren, Member, IEEE, and Ziyang Meng
}

\begin{abstract}
This brief studies distributed containment control for double-integrator dynamics in the presence of both stationary and dynamic leaders. In the case of stationary leaders, we propose a distributed containment control algorithm and study conditions on the network topology and the control gains to guarantee asymptotic containment control in any dimensional space. In the case of dynamic leaders, we study two cases: leaders with an identical velocity and leaders with nonidentical velocities. For the first case, we propose two distributed containment control algorithms to solve, respectively, asymptotic containment control under a switching directed network topology and finite-time containment control under a fixed directed network topology. In particular, asymptotic containment control can be achieved for any dimensional space if the network topology is fixed and for only the 1-D space if the network topology is switching. For the second case, we propose a distributed containment control algorithm under a fixed network topology where the communication patterns among the followers are undirected and derive conditions on the network topology and the control gains to guarantee asymptotic containment control for any dimensional space. Both simulation results and experimental results on a multi-robot platform are provided to validate some theoretical results.
\end{abstract}

Index Terms-Consensus, Containment control, cooperative control, multi-agent systems.

\section{INTRODUCTION}

D ISTRIBUTED cooperative control has been an active research area in the systems and controls society due to its potential applications in both military and civilian sectors. Various distributed cooperative control scenarios have been investigated, including consensus [1]-[3], formation control [4]-[6], rendezvous [7], [8], and flocking [9]-[11].

A fundamental problem investigated in distributed cooperative control is consensus (also called agreement or synchronization in different applications). Consensus means the agreement of a group of vehicles on certain features through only local interaction. Consensus algorithms have been studied extensively for both single-integrator kinematics and double-integrator dynamics. For detailed information about recent study of consensus algorithms, refer to [12], [13], and references therein.

Consensus algorithms were primarily studied when there is no group reference state. Motivated by the fact that a group of

Manuscript received January 29, 2010; revised April 12, 2010; accepted June 06, 2010. Manuscript received in final form June 13, 2010. Date of publication August 03, 2010; date of current version June 17, 2011. Recommended by Associate Editor F. Caccavale. This work was supported by the National Science Foundation under Grant ECCS-1002393.

The authors are with the Department of Electrical and Computer Engineering, Utah State University, Logan, UT 84322 USA (e-mail: wei.ren@usu.edu).

Color versions of one or more of the figures in this brief are available online at http://ieeexplore.ieee.org.

Digital Object Identifier 10.1109/TCST.2010.2053542 vehicles might have a desired trajectory representing the state of interest for the group, consensus in the presence of a group reference state, also called consensus tracking or coordinated tracking, was also investigated. In [14] and [15], a consensus tracking algorithm was studied under a variable undirected network topology. However, [14] and [15] require the availability of the leader's acceleration input to all followers and/or the design of distributed observers. In [16] and [17], a PD-like consensus tracking algorithm was studied in continuous-time and discrete-time settings. However, [16] and [17] require either the availability of the leader's velocity and the followers' velocities or a small sampling period. Consensus tracking in the presence of time-varying delays was investigated in [18]. However, [18] requires the velocity measurements of the followers and the estimation of the leader's velocity.

In containment control problems, there exist multiple leaders and followers and the followers will move in the convex hull spanned by the leaders. Containment control algorithms are consensus-like algorithms in the presence of multiple leaders. The study of containment control is motivated by numerous potential applications. For example, a group of heterogenous vehicles moves from one target to another when only a portion of the vehicles is equipped with necessary sensors to detect the hazardous obstacles. Those vehicles equipped with sensors are normally designated as "leaders" while the other vehicles are designated as "followers." By detecting the positions of the dangerous obstacles, the leaders can form a (moving) safety area. Then the group can arrive at the destination safely given that the followers always stay within the moving safety area formed by the leaders. In [19], a stop-and-go strategy was proposed to drive a group of single-integrator agents to the convex hull spanned by the leaders under a fixed undirected network topology. In [20], containment control for single-integrator kinematics was studied in the presence of both stationary and dynamic leaders under directed switching network topologies. Note that both [19] and [20] focus on single-integrator kinematics.

Taking into account the fact that a broad class of vehicles requires a double-integrator dynamic model, i.e., mass-force model, we study, in this brief, the distributed containment control for double-integrator dynamics in the presence of both stationary and dynamic leaders by expanding on our preliminary work reported in [21]. With multiple stationary leaders, we propose a distributed containment control algorithm and present conditions on the network topology and the control gains to guarantee asymptotic containment control for any dimensional space. With multiple dynamic leaders, we study two cases: leaders with an identical velocity and leaders with nonidentical velocities. For the first case, we propose two distributed containment control algorithms to solve, respectively, asymptotic containment control under a switching directed 
network topology and finite-time containment control under a fixed directed network topology. In particular, when the network topology is fixed, asymptotic containment control can be achieved for any dimensional space. When the network topology is switching, asymptotic containment control can be achieved for only the 1-D space and all followers will converge to the minimal hyperrectangle a high-dimensional space. For the second case, we propose a distributed containment control algorithm and derive conditions on the network topology and the control gains to guarantee asymptotic containment control for any dimensional space under a fixed network topology where the communication patterns among the followers are undirected. Both simulation results and experimental results on a multi-robot platform are provided to validate some theoretical results in this brief. In contrast to many existing results on distributed cooperative control where only theoretical results were provided, we also provide experimental results to validate some theoretical results.

\section{PRELIMINARIES}

\section{A. Graph Theory Notions}

For a group of $n$ vehicles, the interaction for all vehicles can be naturally modeled by a directed graph $\mathcal{G}=(\mathcal{V}, \mathcal{W})$, where $\mathcal{V}=\left\{v_{1}, v_{2}, \ldots, v_{n}\right\}$ and $\mathcal{W} \subseteq \mathcal{V}^{2}$ represent, respectively, the vehicle set and the edge set. Each edge denoted as $\left(v_{i}, v_{j}\right)$ means that vehicle $j$ can access the state information of vehicle $i$, but not necessarily vice versa. Accordingly, vehicle $i$ is a neighbor of vehicle $j$. An edge is undirected if $\left(v_{i}, v_{j}\right) \in \mathcal{W}$ implies $\left(v_{j}, v_{i}\right) \in \mathcal{W}$. We use $\mathcal{N}_{j}$ to denote the neighbor set of vehicle $j$. A directed path is a sequence of edges in a directed graph of the form $\left(v_{1}, v_{2}\right),\left(v_{2}, v_{3}\right), \ldots$, where $v_{i} \in \mathcal{V}$. A directed graph has a directed spanning tree if there exists at least one vehicle that has a directed path to any other vehicle. The union of a set of directed graphs $\mathcal{G}_{i_{1}}, \ldots, \mathcal{G}_{i_{m}}$ is a directed graph with the edge set given by the union of the edge sets of the directed graphs $\mathcal{G}_{i_{j}}, j=1, \ldots, m$, where $\mathcal{G}_{i_{j}}, j=1, \ldots, m$, have the same vehicle set.

The interaction graph can be mathematically represented by two matrices: the adjacency matrix $\mathcal{A}=\left[a_{i j}\right] \in \mathbb{R}^{n \times n}$ with $a_{i j}>0$ if $\left(v_{j}, v_{i}\right) \in \mathcal{W}$ and $a_{i j}=0$ otherwise, and the (non-symmetric) Laplacian matrix $\mathcal{L}=\left[\ell_{i j}\right] \in \mathbb{R}^{n \times n}$ with $\ell_{i i}=\sum_{j=1, j \neq i}^{n} a_{i j}$ and $\ell_{i j}=-a_{i j}, i \neq j$. Here we assume that $a_{i i}=0, \forall i=1, \ldots, n$. For undirected graphs, we assume that $a_{i j}=a_{j i}$. It is easy to verify that $\mathcal{L}$ has at least one zero eigenvalue with a corresponding eigenvector $\mathbf{1}$, where $\mathbf{1}$ is an all-one column vector with a compatible size.

\section{B. Definitions}

The following definitions are borrowed from [20].

Definition 2.1: For a group of $n$ vehicles, a vehicle is called a leader if the vehicle has no neighbor. A vehicle is called a follower if the vehicle has a neighbor. Assume that there are $m$ followers, where $m<n$. We use $\mathcal{R}$ and $\mathcal{F}$ to denote, respectively, the leader set and the follower set. The directed graph $\mathcal{G}$ has a united directed spanning tree if for any one of the $m$ followers, there exists at least one leader that has a directed path to the follower.
Definition 2.2: Let $\mathcal{C}$ be a set in a real vector space $V \subseteq \mathbb{R}^{p}$. The set $\mathcal{C}$ is called convex if, for any $x$ and $y$ in $\mathcal{C}$, the point $(1-z) x+z y$ is in $\mathcal{C}$ for any $z \in[0,1]$. The convex hull for a set of points $X=\left\{x_{1}, \ldots, x_{q}\right\}$ in $V$ is the minimal convex set containing all points in $X$. We use $\operatorname{Co}\left\{x_{i}, i=1, \ldots, q\right\}$ to denote the convex hull of $X$. In particular, when $V \subseteq \mathbb{R}, \mathbf{C o}\left\{x_{i}, i=\right.$ $1, \ldots, q\}=\left\{x \mid x \in\left[\min _{i} x_{i}, \max _{i} x_{i}\right]\right\}$.

\section{Stability Analysis With Multiple STATIONARY LEADERS}

In this section, we study the conditions on, respectively, the fixed and switching network topologies such that all followers will converge to the stationary convex hull formed by the stationary leaders. That is, all followers will ultimately move into the convex hull formed by the stationary leaders for arbitrary initial states of the followers.

Consider a group of $n$ vehicles with double-integrator dynamics given by

$$
\dot{x}_{i}(t)=v_{i}(t), \quad \dot{v}_{i}(t)=u_{i}(t) \quad i=1, \ldots, n
$$

where $x_{i}(t) \in \mathbb{R}^{p}, v_{i}(t) \in \mathbb{R}^{p}$, and $u_{i}(t) \in \mathbb{R}^{p}$ are, respectively, the position, the velocity, and the control input associated with the $i$ th vehicle. We propose the following containment control algorithm for (1) as:

$$
\begin{aligned}
v_{i}(t)= & 0, \quad i \in \mathcal{R}, \\
u_{i}(t)= & -\beta v_{i}(t)-\sum_{j \in \mathcal{F} \bigcup \mathcal{R}} a_{i j}(t) \\
& \times\left\{\beta\left[x_{i}(t)-x_{j}(t)\right]+\left[v_{i}(t)-v_{j}(t)\right]\right\}, \quad i \in \mathcal{F}
\end{aligned}
$$

where $\mathcal{R}$ and $\mathcal{F}$ are defined in Definition $2.1, a_{i j}(t)$ is the $(i, j)$ th entry of the adjacency matrix $\mathcal{A}$ at time $t$, and $\beta$ is a positive constant. The objective of (2) is to guarantee that all vehicles move into the convex hull formed by the leaders. Note that $x_{j}(t), j \in \mathcal{R}$, is constant because the leaders are stationary.

We assume that $\mathcal{A}(t)$ (i.e., the interaction among the $n$ vehicles) is constant over time intervals $\left[\sum_{j=1}^{k} \Delta_{j}, \sum_{j=1}^{k+1} \Delta_{j}\right)^{1}$ and switches at time $t=\sum_{j=1}^{k} \Delta_{j}$ with $k=0,1, \ldots$, where $\Delta_{j}>0, j=1, \ldots$. Let $\mathcal{G}_{k}$ and $\mathcal{A}[k]$ denote, respectively, the directed graph and the adjacency matrix for the $n$ vehicles for $t \in\left[\sum_{j=1}^{k} \Delta_{j}, \sum_{j=1}^{k+1} \Delta_{j}\right)$. We first consider the case when the vehicles are in a $1-\mathrm{D}$ space [i.e., $p=1$ in (1)].

Theorem 3.1: Using (2) for (1), all followers will always converge to the stationary convex hull $\operatorname{Co}\left\{x_{j}, j \in \mathcal{R}\right\}$ for arbitrary initial conditions $x_{i}(0), i \in \mathcal{F}$, if and only if there exists a positive integer $N_{2}$ such that the union of $\mathcal{G}_{i}, i=N_{1}, \ldots, N_{1}+N_{2}$, has a united directed spanning tree for any finite $N_{1}$.

Proof: (Necessity) We prove this part by contraposition motivated by [22]. Assume that for every positive integer $N_{2}$, there exists positive $N_{1}$ such that the union of $G_{i}, i=N_{1}, \ldots, N_{1}+N_{2}$, does not have a united directed spanning tree. This implies that for every positive integer $N_{2}$, there exists positive $N_{1}$ such that at least one follower, labeled as $F_{m}$, who cannot access any leader's state directly or indirectly for $t \in\left[\sum_{j=1}^{N_{1}} \Delta_{j}, \sum_{j=1}^{N_{1}+N_{2}} \Delta_{j}\right.$ ). Because $N_{2}$ can be chosen arbitrarily large, it is straightforward to show that the

\footnotetext{
${ }^{1}$ When $k=0$, we define $\sum_{j=1}^{k} \Delta_{j} \triangleq 0$.
} 
state of $F_{m}$ will not be affected by the leaders' states for any $t \geq \sum_{j=1}^{N_{1}} \Delta_{j}$. That is, $F_{m}$, in general, cannot always converge to the stationary convex hull $\operatorname{Co}\left\{x_{j}, j \in \mathcal{R}\right\}$ for arbitrary initial conditions.

(Sufficiency) Define $z_{i}(t) \triangleq \beta x_{i}(t)+v_{i}(t), i=1, \ldots, n$. Using (2) for (1), we can get that

$$
\begin{aligned}
& \dot{z}_{i}(t)=0, i \in \mathcal{R}, \\
& \dot{z}_{i}(t)=-\sum_{j \in \mathcal{R} \bigcup \mathcal{F}} a_{i j}[k]\left[z_{i}(t)-z_{j}(t)\right], \quad i \in \mathcal{F} .
\end{aligned}
$$

By following a similar analysis to that in the proof of Theorem 3.1 in [20], it can be shown that $z_{i}(t), i \in \mathcal{F}$, will converge to the convex hull $\operatorname{Co}\left\{z_{j}, j \in \mathcal{R}\right\}$ under the condition of the theorem. Define $\bar{z} \triangleq \max _{i \in \mathcal{R}} z_{i}, \bar{x} \triangleq \max _{i \in \mathcal{R}} x_{i}$ and $\delta_{i} \triangleq x_{i}-\bar{x}, i \in \mathcal{F}$. Here $\bar{z}$ and $\bar{x}$ are constant because the leaders are stationary. Note that $z_{i}(t)-\bar{z}(t)$ is bounded and $z_{i}(t)-\bar{z}(t) \leq 0$ as $t \rightarrow \infty$. Letting $f_{i}(t)=z_{i}(t)-\bar{z}(t)$, it then follows that

$$
\dot{\delta}_{i}(t)+\beta \delta_{i}(t)=f_{i}(t)
$$

where we have used the fact that $x_{i}(t), i \in \mathcal{R}$, are constant. Therefore, the solution of (4) is given by $\delta_{i}(t)=e^{-\beta t} \delta_{i}(0)+$ $\int_{0}^{t} e^{-\beta(t-\tau)} f_{i}(\tau) d \tau$. It follows that

$$
\begin{aligned}
\lim _{t \rightarrow \infty} \delta_{i}(t) & =\lim _{t \rightarrow \infty} e^{-\beta t} \delta_{i}(0)+\lim _{t \rightarrow \infty} \int_{0}^{t} e^{-\beta(t-\tau)} f_{i}(\tau) d \tau \\
& =\lim _{t \rightarrow \infty} \frac{\int_{0}^{t} e^{\beta \tau} f_{i}(\tau) d \tau}{e^{\beta t}} \\
& =\lim _{t \rightarrow \infty} \frac{e^{\beta t} f_{i}(t)}{\beta e^{\beta t}} \\
& =\lim _{t \rightarrow \infty} \frac{f_{i}(t)}{\beta} .
\end{aligned}
$$

Noting that $\beta>0$ and $f_{i}(t) \leq 0$ as $t \rightarrow \infty$, it then follows that $\delta_{i}(t) \leq 0$ as $t \rightarrow \infty$, i.e., $x_{i}(t) \leq \bar{x}(t)$ as $t \rightarrow \infty$. This implies that $x_{i}(t) \leq \max _{j \in \mathcal{R}} x_{j}(t)$ as $t \rightarrow \infty$. Similarly, it can be shown that $x_{i}(t) \geq \min _{j \in \mathcal{R}} x_{j}(t)$ as $t \rightarrow \infty$. Therefore, all followers will converge to the convex hull formed by the leaders.

Remark 3.2: In Theorem 3.1, all followers are shown to converge to the convex hull in a 1-D space. For any high-dimensional space, by using the decoupling technique, it is straightforward to show that all followers will converge to the minimal hyperrectangle that contains the stationary leaders and each of whose hyperplanes is normal to one axis of a chosen inertial coordinate frame. Note that the closed-loop system by using (2) for (1) is independent of the inertial coordinate frame that is chosen. Therefore, the followers will converge to the intersection of all minimal hyperrectangles that contain the leaders. Note also that the intersection of all minimal hyperrectangles that contain the leaders is essentially the convex hull formed by the leaders. Therefore, all followers will converge to the convex hull formed by the leaders in any high-dimensional space under the condition of Theorem 3.1.

\section{Stability ANAlysis With Multiple Dynamic Leaders}

In Section III, the leaders are assumed to be stationary. In this section, we study distributed containment control for double-in- tegrator dynamics in the presence of multiple dynamic leaders. We consider two cases: leaders with an identical velocity and leaders with nonidentical velocities.

\section{A. Leaders With an Identical Velocity}

In this subsection, we assume that the velocities of all leaders are the same (i.e., $v_{i}(t)=v_{j}(t)$ for $i, j \in \mathcal{R}$ ).

For (1), we propose the following containment control algorithm as:

$$
\begin{aligned}
v_{i}(t)=v^{o}(t), \quad i \in \mathcal{R}, & u_{i}(t)=-\gamma \operatorname{sgn}\left(\sum _ { j \in \mathcal { F } \bigcup \mathcal { R } } a _ { i j } ( t ) \left\{\beta\left[x_{i}(t)-x_{j}(t)\right]\right.\right. \\
& \left.\left.+\left[v_{i}(t)-v_{j}(t)\right]\right\}\right)-\beta v_{i}(t), \quad i \in \mathcal{F}
\end{aligned}
$$

where $v^{o}(t)$ is the common velocity of the leaders, $\beta$ and $\gamma$ are positive constants, and $\operatorname{sgn}(\cdot)$ is the signum function defined entrywise. Note that the right-hand side of (5) [correspondingly, (8) and (9)] is discontinuous. Therefore, the solution is studied in terms of differential inclusions [23], [24]. We first study the case when the vehicles are in a 1-D space.

Theorem 4.1: Assume that the network topology switches according to the same model as described right before Theorem 3.1. Assume also that $\gamma>\gamma_{\ell}$, where $\gamma_{\ell} \triangleq \sup _{i \in \mathcal{R}} \mid \dot{v}^{o}(t)+$ $\beta v^{o}(t) \mid$ is bounded. All followers will always converge to the dynamic convex hull $\mathbf{C o}\left\{x_{j}, j \in \mathcal{R}\right\}$ for arbitrary initial conditions $x_{i}(0), i \in \mathcal{F}$, if the network topology has a united directed spanning tree at each time interval.

Proof: Define $z_{i}(t) \triangleq \beta x_{i}(t)+v_{i}(t)$. Using (5) for (1) gives

$$
\begin{aligned}
& \dot{z}_{i}(t)=\dot{v}^{o}(t)+\beta v^{o}(t), \quad i \in \mathcal{R}, \\
& \dot{z}_{i}(t)=-\gamma \mathrm{sgn}\left\{\sum_{j \in \mathcal{R} \bigcup \mathcal{F}} a_{i j}[k]\left[z_{i}(t)-z_{j}(t)\right]\right\}, i \in \mathcal{F} .
\end{aligned}
$$

We next show that $z_{i}(t)$ will converge to the convex hull $\operatorname{Co}\left\{z_{j}, j \in \mathcal{R}\right\}$ in finite time.

Define $\bar{z} \triangleq \max _{i \in \mathcal{R}} z_{i}, \underline{z} \triangleq \min _{i \in \mathcal{R}} z_{i}, \tilde{z} \triangleq \max _{i \in \mathcal{F}} z_{i}-\bar{z}$, and $\breve{z} \triangleq \min _{i \in \mathcal{F}} z_{i}-\underline{z}$. Noting that the leaders have the same velocity, if $x_{i}(0)>x_{j}(0)$, then $x_{i}(t)>x_{j}(t)$ for any $t>0$. This implies that if leader $L_{m}$ has the maximal state at $t=0$, leader $L_{m}$ always has the maximal state, i.e., $\bar{z}=\beta x_{m}(t)+v_{m}(t)$. Therefore, $\bar{z}$ is differentiable and the derivative of $\bar{z}$ is equal to $\dot{v}^{o}(t)+\beta v^{o}(t)$. Similarly, it can be shown that $\underline{z}$ is differentiable and the derivative of $\underline{z}$ is also equal to $\dot{v}^{o}(t)+\beta v^{o}(t)$. We next will show that $\tilde{z}(t) \leq 0$ and $\breve{z}(t) \geq 0$ in finite time. Here we only consider the case when $\tilde{z}(0)>0$ and $\breve{z}(0) \geq 0$. Similar analysis can also be applied to other cases. We next show that when $\tilde{z}(t)>0$ for $t \leq T$ with $T>0, \dot{\tilde{z}}(t) \leq-\gamma+\gamma_{\ell}$ for $t \leq T$ except for some isolated time instants. We prove this by contradiction. Because $\tilde{z}(t)>0$ for $t \leq T$, it follows from (6) and the definition of $\tilde{z}$ that $\dot{\tilde{z}}(t)=-\dot{\bar{z}}(t)$ or $\dot{\tilde{z}}(t)<-\gamma+\gamma_{\ell}$ for $t \leq T$. Assume that $\dot{\tilde{z}}(t)=-\dot{\bar{z}}(t)$ for $t \in\left[t_{1}, t_{2}\right]$, where $t_{1}<t_{2} \leq T$. 
There exists some vehicle, labeled $j$, with the maximal state satisfying $\dot{z}_{j}(t)=-\dot{\bar{z}}(t)$ for $t \in\left[t_{1}, t_{3}\right]$, where $t_{1}<t_{3} \leq t_{2}$. It then follows from (6) that $\sum_{i \in \mathcal{R}} \bigcup \mathcal{F} a_{j i}[k]\left[z_{j}(t)-z_{i}(t)\right]=0$ for $t \in\left[t_{1}, t_{3}\right]$. Because vehicle $j$ is with the maximal state, it then follows that $z_{i}(t)=z_{j}(t), \forall i \in \mathcal{N}_{j}$, for $t \in\left[t_{1}, t_{3}\right]$. By following a similar analysis, when $\mathcal{G}$ has a united directed spanning tree at each time interval, $z_{\kappa}(t)=z_{j}(t)$ for some $\kappa \in \mathcal{R}$ for $t \in\left[t_{1}, t_{3}\right]$, which results in a contradiction because $\tilde{z}(T)>0$. Therefore, $\tilde{z}(t)$ will keep decreasing with a speed large than $\gamma-\gamma_{\ell}$ for $t \leq T$ except for some isolated time instants. From the proof of Theorem 4.2 in [20], if $\tilde{z}\left(t_{1}\right) \leq 0$, then $\tilde{z}(t) \leq 0$ for $t \geq t_{1}$. It then follows that $\tilde{z}(t) \leq 0$ in finite time. Similarly, it can be shown that $\breve{z}(t) \geq 0$ in finite time. Therefore, $z_{i}(t), i \in \mathcal{F}$, will converge to the convex hull $\mathbf{C o}\left\{z_{j}, j \in \mathcal{R}\right\}$ in finite time.

Because $z_{i}(t), i \in \mathcal{F}$, will converge to the convex hull $\operatorname{Co}\left\{z_{j}, j \in \mathcal{R}\right\}$ in finite time, there exists positive $\bar{t}$ such that $\min _{j \in \mathcal{R}} z_{j}(t) \leq z_{i}(t) \leq \max _{j \in \mathcal{R}} z_{j}(t), i \in \mathcal{F}$, for $t \geq \bar{t}$. Because the leaders have the same velocity, if $x_{i}(0) \geq x_{j}(0), \forall i, j \in \mathcal{R}$, then $x_{i}(t) \geq x_{j}(t)$ for any $t>0$, which implies that $z_{i}(t) \geq z_{j}(t)$ for any $t>0$. Without loss of generality, let $\max _{j \in \mathcal{R}} z_{j}(t)=\beta x_{p}(t)+\dot{x}_{p}(t)$ for some $p \in \mathcal{R}$ and $\min _{j \in \mathcal{R}} z_{j}(t)=\beta x_{q}(t)+\dot{x}_{q}(t)$ for some $q \in \mathcal{R}$. It then follows that $\beta x_{q}(t)+\dot{x}_{q}(t) \leq \beta x_{i}(t)+\dot{x}_{i}(t) \leq \beta x_{p}(t)+\dot{x}_{p}(t)$, $i \in \mathcal{F}$, for $t \geq \bar{t}$. For $t \geq \bar{t}$ and $i \in \mathcal{F}$

$$
\begin{aligned}
& \dot{x}_{i}(t)-\dot{x}_{q}(t) \geq-\beta\left[x_{i}(t)-x_{q}(t)\right] \\
& \dot{x}_{i}(t)-\dot{x}_{p}(t) \leq-\beta\left[x_{i}(t)-x_{p}(t)\right] .
\end{aligned}
$$

Therefore, $x_{q}(t) \leq x_{i}(t) \leq x_{p}(t), i \in \mathcal{F}$, as $t \rightarrow \infty$. Because the leaders have the same velocity, all followers will converge to the convex hull formed by the leaders.

Remark 4.2: When the directed network topology is fixed and the vehicles are in a 1-D space, by following a similar analysis to that of Theorem 4.1 in [20], it can be shown that $\mathcal{L} Z(t) \rightarrow \mathbf{0}$ as $t \rightarrow \infty$, where $Z(t)=\left[z_{1}(t), \ldots, z_{n}(t)\right]^{T}$ and $\mathbf{0}$ is an all-zero vector with a compatible size, under the condition of Theorem 4.1. Noting that all leaders have the same velocity, it is easy to show that $\mathcal{L} X(t) \rightarrow \mathbf{0}$ as $t \rightarrow \infty$, where $X(t)=\left[x_{1}(t), \ldots, x_{n}(t)\right]^{T}$. Similarly, when the vehicles are in any high-dimensional space [i.e., $p>1$ in (1)], by using the decoupling technique, it can be shown that $\left(\mathcal{L} \otimes I_{p}\right) X(t) \rightarrow \mathbf{0}$ as $t \rightarrow \infty$, where $\otimes$ is the Kronecker product. Therefore, all followers will converge to the convex hull formed by the leaders for any high-dimensional space under a fixed directed network topology when the conditions of Theorem 4.1 are satisfied. In contrary, when the switching directed network topology has a directed spanning tree at each time interval and the vehicles are in a high-dimensional space, by using the decoupling technique, it can be shown that the followers will converge to the minimal interval containing the leaders in each dimension. That is, the followers will converge to the minimal hyperrectangle that contains the leaders and each of whose hyperplanes is normal to one axis of the chosen coordinate frame. Meanwhile, because the network topology might change as $t$ increases, $\left(\mathcal{L}(t) \otimes I_{p}\right) X(t)$ might not approach $\mathbf{0}$ as $t \rightarrow \infty$. Therefore the followers might not be able to converge to the convex hull formed by the leaders under switching topologies in a high-dimensional space. Note that the changing sequence of the network topology plays an important role in determining whether the followers will ultimately converge to the convex hull.

We next propose a distributed containment control algorithm which can guarantee that all followers converge to the convex hull formed by the leaders in finite time. In the following of this subsection and Section IV-B, we assume that all vehicles are in a 1-D space for the simplicity of presentation. However, all the results hereafter are still valid for arbitrary high-dimensional space by using the decoupling technique and the Kronecker product.

Inspired by [25], we propose the following finite-time containment control algorithm as:

$$
\begin{aligned}
u_{i}(t)=\dot{\hat{v}}_{i}(t)-\operatorname{sgn}\left[v_{i}(t)-\hat{v}_{i}(t)\right] \mid & v_{i}(t)-\left.\hat{v}_{i}(t)\right|^{\kappa} \\
& -\operatorname{sgn}[\phi(t)]|\phi(t)|^{\frac{\kappa}{2-\kappa}}
\end{aligned}
$$

where $0<\kappa<1, \phi(t)=x_{i}(t)-\hat{x}_{i}(t)+$ $(1) /(2-\kappa) \operatorname{sgn}\left[v_{i}(t)-\hat{v}_{i}(t)\right]\left|v_{i}(t)-\hat{v}_{i}(t)\right|^{2-\kappa}$, and

$$
\begin{aligned}
& \dot{\hat{x}}_{i}(t)=\hat{v}_{i}(t)-\rho_{1} \operatorname{sgn}\left\{\sum_{j \in \mathcal{R} \bigcup \mathcal{F}} a_{i j}\left[\hat{x}_{i}(t)-\hat{x}_{j}(t)\right]\right\} \\
& \dot{\hat{v}}_{i}(t)=-\rho_{2} \operatorname{sgn}\left\{\sum_{j \in \mathcal{R} \bigcup \mathcal{F}} a_{i j}\left[\hat{v}_{i}(t)-\hat{v}_{j}(t)\right]\right\}, i \in \mathcal{F}
\end{aligned}
$$

with $\hat{x}_{i}(t) \triangleq x_{i}(t)$ and $\hat{v}_{i}(t)=v^{o}(t)$ for $i \in \mathcal{R}$.

Theorem 4.3: Assume that the fixed network topology has a united directed spanning tree and $\sup _{t}\left|\dot{v}^{o}(t)\right|$. Using (7) for (1), all followers will converge to the dynamic convex hull formed by the leaders in finite time if $\rho_{1}>0$ and $\rho_{2}>\sup _{t}\left|\dot{v}^{o}(t)\right|$.

Proof: When $\rho_{2}>\sup _{t}\left|\dot{v}^{o}(t)\right|$, it follows from (9) and a similar proof to that of Theorem 4.1 that $\hat{v}_{i}(t), i \in \mathcal{F}$, will converge to the convex hull formed by the leaders in finite time. Noting that $\hat{v}_{j}(t) \triangleq v^{o}(t), \forall j \in \mathcal{R}$, it follows that $\hat{v}_{i}(t)=$ $v^{o}(t), i \in \mathcal{F} \cup \mathcal{R}$, in finite time. Without loss of generality, let $\hat{v}_{i}(t)=v^{o}(t), i \in \mathcal{F} \cup \mathcal{R}$, for $t \geq \bar{t}_{1}$, where $\bar{t}_{1}$ is some positive constant. For $t \geq \bar{t}_{1}$, (8) can be written in matrix form as

$$
\dot{\hat{x}}(t)=v^{o}(t) \mathbf{1}-\rho_{1} \operatorname{sgn}[\mathcal{L} \hat{x}(t)]
$$

where $\hat{x}(t)=\left[\hat{x}_{1}(t), \ldots, \hat{x}_{n}(t)\right]^{T}$ and $\mathcal{L}$ is the Laplacian matrix of the $n$ vehicles including both the leaders and the followers. Let $z(t)=\left[z_{1}(t), \ldots, z_{n}(t)\right]^{T}=\mathcal{L} \hat{x}(t)$. Because $\mathcal{L} 1=0$, it follows that (10) can be rewritten as $\dot{z}(t)=-\rho_{1} \mathcal{L} \operatorname{sgn}[z(t)]$. Note that $z_{i}(t)=0, i \in \mathcal{R}$, because the $i$ th row of $\mathcal{L}$ is zero. When the fixed graph has a united directed spanning tree, it can be shown that $z_{i}(t) \rightarrow 0$ in finite time by showing that both the maximal and minimal states will go to zero in finite time. This implies that $\hat{x}_{i}(t), i \in \mathcal{F}$, will be within the convex hull formed by the leaders in finite time. It then follows from (10) that $\dot{\hat{x}}_{i}(t) \rightarrow v^{o}(t)$ in finite time. Without loss of generality, let $\dot{\hat{x}}_{i}(t)=v^{o}(t)$ for $t \geq \bar{t}_{2}$, where $\bar{t}_{2}$ is some positive constant. Note also that $\dot{\hat{v}}_{i}(t)$ can be replaced with $\dot{v}^{o}(t)$ for $t \geq \bar{t}_{2}$ because $\int_{t_{1}}^{t_{2}} \dot{\hat{v}}_{i}(t) d t=\int_{t_{1}}^{t_{2}} \dot{v}_{i}^{o}(t) d t$ for any $t_{2}>t_{1} \geq \bar{t}_{2}$. 


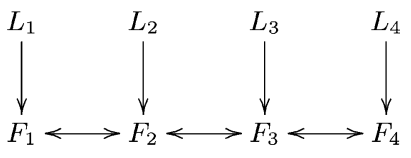

(a)

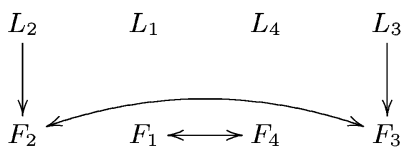

(b)

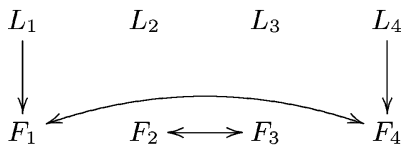

(c)

Fig. 1. Network topology for a group of vehicles with multiple leaders. $L_{i}, i=$ $1, \ldots, 4$, denote the leaders. $F_{i}, i=1, \ldots, 6$, denote the followers. (a) Graph 1. (b) Graph 2. (c) Graph 3.

Define $\delta_{i} \triangleq x_{i}(t)-\hat{x}_{i}(t)$. For $t \geq \bar{t}_{2}$, by replacing $\dot{\hat{v}}_{i}(t)$ with $\dot{v}^{o}(t),(7)$ can be rewritten as $\ddot{\delta}_{i}(t)=-\operatorname{sgn}\left[\dot{\delta}_{i}(t)\right]\left|\dot{\delta}_{i}(t)\right|^{\kappa}-$ $\operatorname{sgn}\left[\phi_{i}(t)\right]\left|\phi_{i}(t)\right|^{(\kappa) /(2-\kappa)}$. It then follows from [25, Proposition 1] that $\delta_{i}(t) \rightarrow 0$ in finite time.

Combining the previous statements completes the proof.

\section{B. Leaders With Nonidentical Velocities}

In this subsection, we consider a more general case where the velocities of the leaders are nonidentical. Without loss of generality, we assume that the first $n-m$ vehicles are leaders.

For (1), we propose the following containment control algorithm as:

$$
\begin{aligned}
u_{i}(t)= & a_{i}(t), \quad i=1, \ldots, n-m \\
u_{i}(t)= & -\sum_{j \in \mathcal{R} \bigcup \mathcal{F}} a_{i j}\left[\left(x_{i}-x_{j}\right)+\alpha\left(v_{i}-v_{j}\right)\right] \\
& -\beta \operatorname{sgn}\left\{\sum_{\left.j \in \mathcal{R} \bigcup \mathcal{F} \quad a_{i j}\left[\gamma\left(x_{i}-x_{j}\right)+\left(v_{i}-v_{j}\right)\right]\right\}}^{i=n-m+1, \ldots, n},\right.
\end{aligned}
$$

where $a_{i}(t)$ is the acceleration input for the $i$ th leader, and $\alpha$, $\beta$, and $\gamma$ are positive constants. Using (11), (1) can be written in matrix form as

$$
\ddot{X}=-\mathcal{L} X-\alpha \mathcal{L} \dot{X}-\beta \operatorname{sgn}(\gamma \mathcal{L} X+\mathcal{L} \dot{X})-\Psi
$$

where $X=\left[x_{1}, \ldots, x_{n}\right]^{T}, \Psi(t)=\left[\psi_{1}(t), \ldots, \psi_{n}(t)\right]^{T}$ with $\psi_{i}(t)=a_{i}(t)$ for $i=1, \ldots, n-m$, and $\psi_{i}(t)=0$ for $i=$ $n-m+1, \ldots, n$, and $\mathcal{L}$ is the Laplacian matrix defined in Section II. Note that the first $n-m$ rows of $\mathcal{L}$ are equal to zero. Define $\tilde{X} \triangleq \mathcal{L} X$. It follows that the first $n-m$ entries of $\tilde{X}$ are equal to zero. Then (12) can be written as

$$
\ddot{\tilde{X}}=-\mathcal{L} \tilde{X}-\alpha \mathcal{L} \dot{\tilde{X}}-\beta \mathcal{L} \operatorname{sgn}(\gamma \tilde{X}+\dot{\tilde{X}})-\mathcal{L} \Psi .
$$

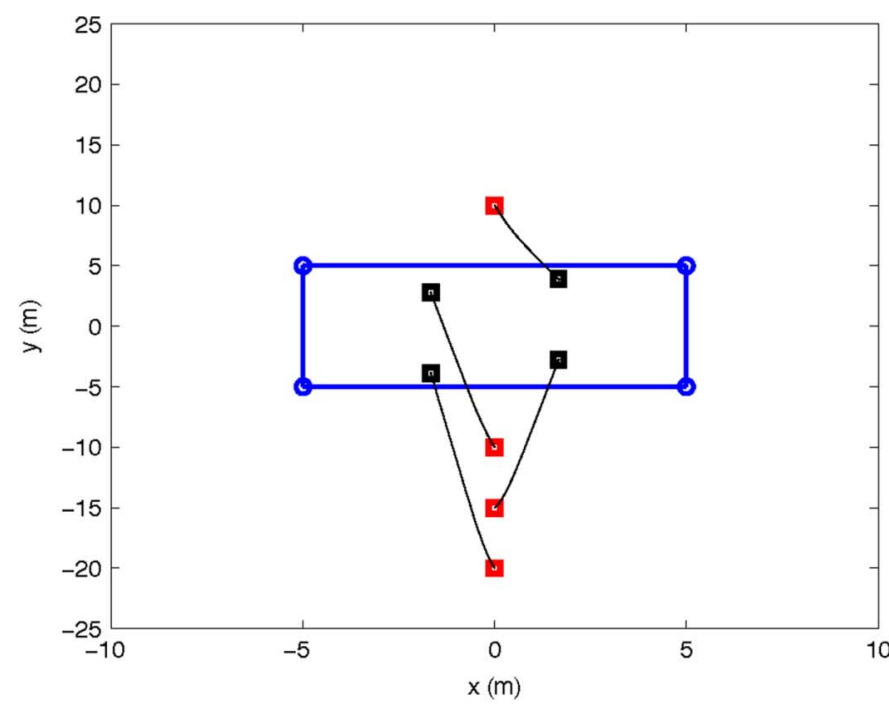

(a)

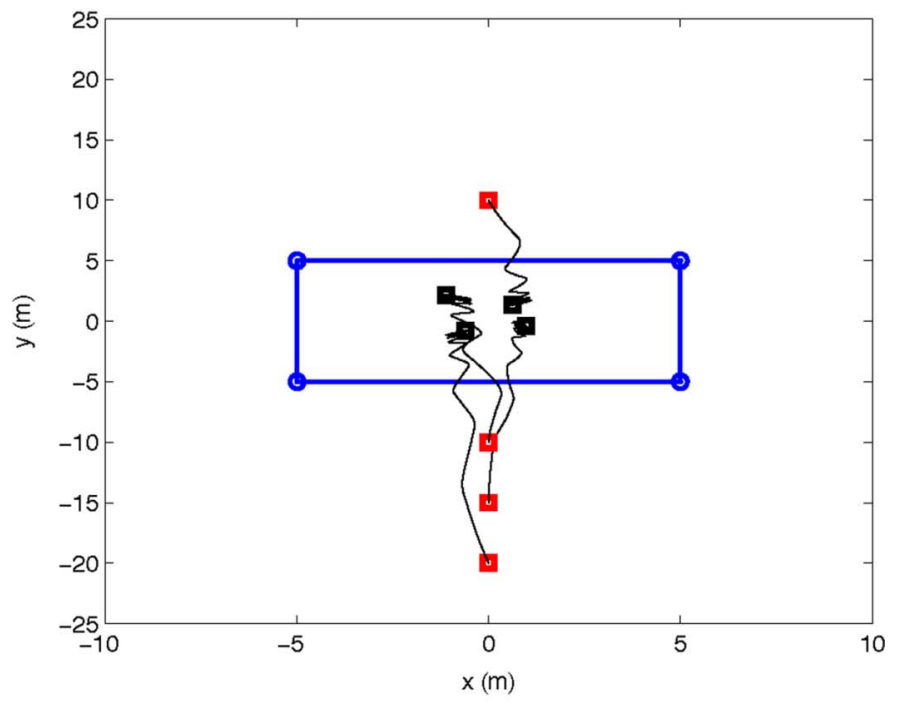

(b)

Fig. 2. Trajectories of the agents using (2) under a fixed and a switching directed network topology in 2-D. Circles denote the starting positions of the stationary leaders while the red and black squares denote, respectively, the starting and ending positions of the followers. (a) Fixed directed network topology. (b) Switching directed network topology.

Let $\tilde{X}_{F}$ be the vector containing only the last $m$ entries of $\tilde{X}$ and $\Psi_{F}$ be the vector containing only the last $m$ entries of $\mathcal{L} \Psi$. Therefore, (14) can be rewritten as

$$
\ddot{\tilde{X}}_{F}=-M \tilde{X}_{F}-\alpha M \dot{\tilde{X}}_{F}-\beta M \operatorname{sgn}\left(\gamma \tilde{X}_{F}+\dot{\tilde{X}}_{F}\right)-\Psi_{F}
$$

where $M=\left[m_{i j}\right] \in \mathbb{R}^{m \times m}$ with $m_{i j}=\ell_{i j}$ if $i \neq j$ and $m_{i i}=\sum_{k \neq i} \ell_{i k}$.

Lemma 4.1: Assume that the fixed graph has a united directed spanning tree and the communication patterns among the followers are undirected. Let $P=\left[\begin{array}{cc}I_{n} & \gamma M^{-1} \\ \gamma M^{-1} & M^{-1}\end{array}\right]$ and $Q=\left[\begin{array}{cc}\gamma I_{n} & (\alpha \gamma) /(2) I_{n} \\ (\alpha \gamma) /(2) I_{n} & \alpha I_{n}-\gamma M^{-1}\end{array}\right]$, where $\gamma$ and $\alpha$ are two positive constants and $M$ is defined right before this lemma. If $\alpha$ and $\gamma$ are chosen satisfying 


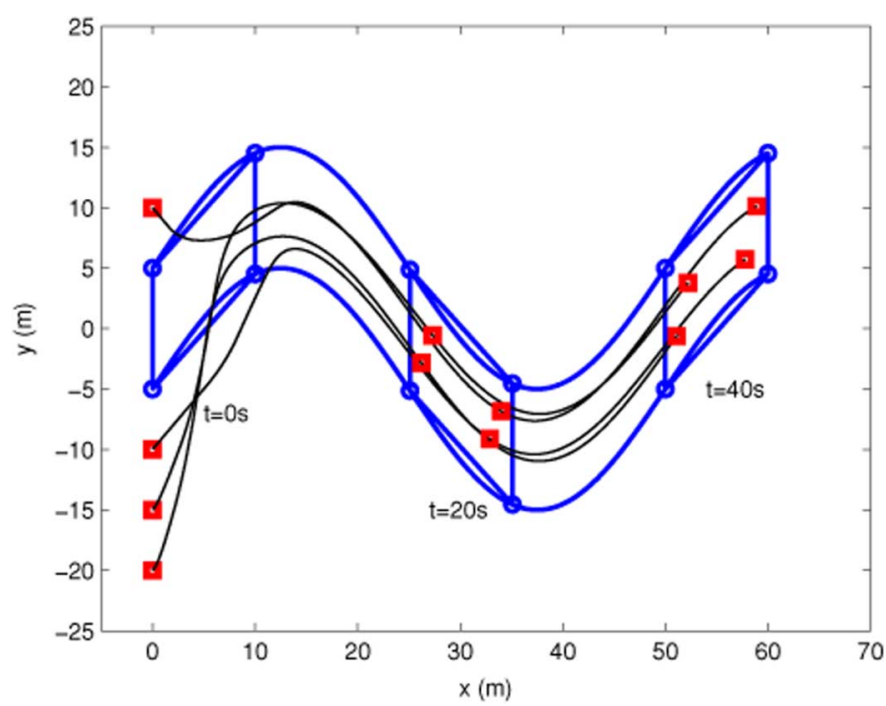

Fig. 3. Trajectories of the agents using (5) under a fixed directed network topology in 2-D. Circles denote the positions of the dynamic leaders while the squares denote the positions of the followers. Two snapshots at $t=25 \mathrm{~s}$ and $t=50 \mathrm{~s}$ show that all followers remain in the dynamic convex hull formed by the dynamic leaders.

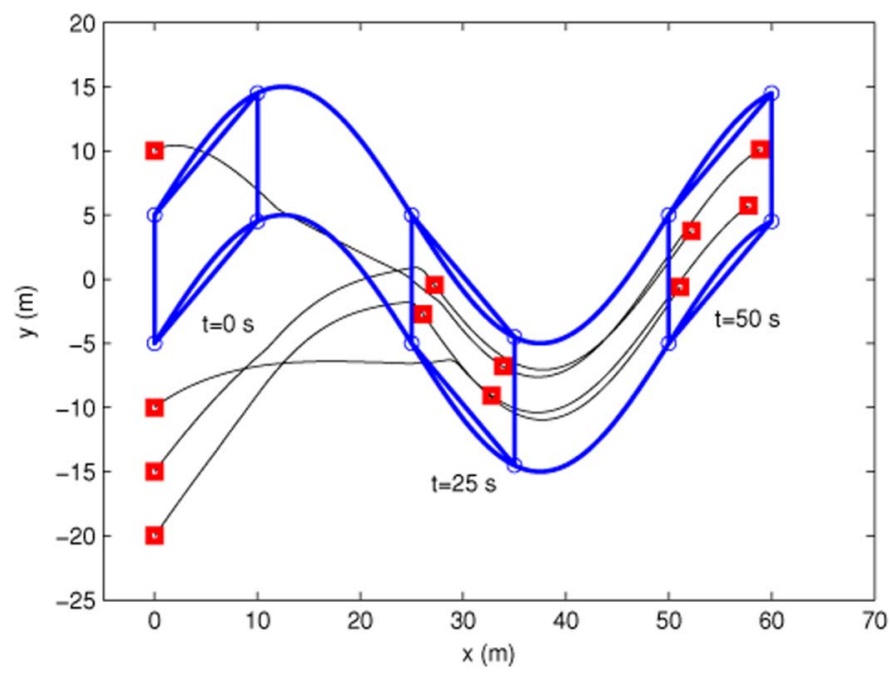

Fig. 4. Trajectories of the vehicles using (7) under a fixed directed network topology in 2-D. Circles denote the positions of the dynamic leaders while the squares denote the positions of the followers. Two snapshots at $t=25 \mathrm{~s}$ and $t=50 \mathrm{~s}$ show that all followers remain in the dynamic convex hull formed by the dynamic leaders.

$\gamma<\min \left\{\sqrt{\lambda_{\min }(M)},\left(4 \alpha \lambda_{\min }(M)\right) /\left(4+\alpha^{2} \lambda_{\min }(M)\right)\right\}$, both $P$ and $Q$ are symmetric positive definite, where $\lambda_{\min }(\cdot)$ denotes the smallest eigenvalue of a symmetric matrix.

Proof: When the graph has a united directed spanning tree and the communication patterns among the followers are undirected, $M$ is symmetric positive definite. Therefore, $M^{-1}$ is also symmetric positive definite. Then $M^{-1}$ can be diagonalized as $M^{-1}=\Gamma^{-1} \Lambda \Gamma$, where $\Lambda=\operatorname{diag}\left\{(1) /\left(\lambda_{1}\right), \ldots,(1) /\left(\lambda_{m}\right)\right\}$ with $\lambda_{i}$ being the $i$ th eigenvalue of $M$. Let $\mu$ be an eigenvalue of $P$. It then follows that $\mu$ satisfies

$$
(\mu-1)\left(\mu-\frac{1}{\lambda_{i}}\right)-\frac{\gamma^{2}}{\lambda_{i}^{2}}=0
$$

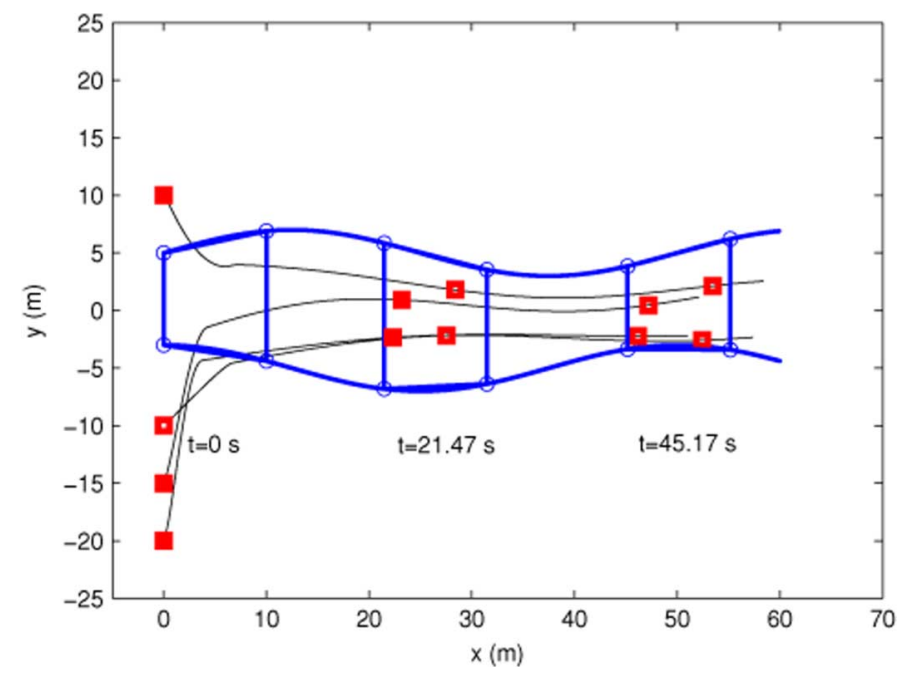

Fig. 5. Trajectories of the vehicles using (11) under a fixed directed network topology in 2-D. Circles denote the positions of the dynamic leaders while the squares denote the positions of the followers. Two snapshots at $t=21.47 \mathrm{~s}$ and $t=45.17 \mathrm{~s}$ show that all followers remain in the dynamic convex hull formed by the dynamic leaders.

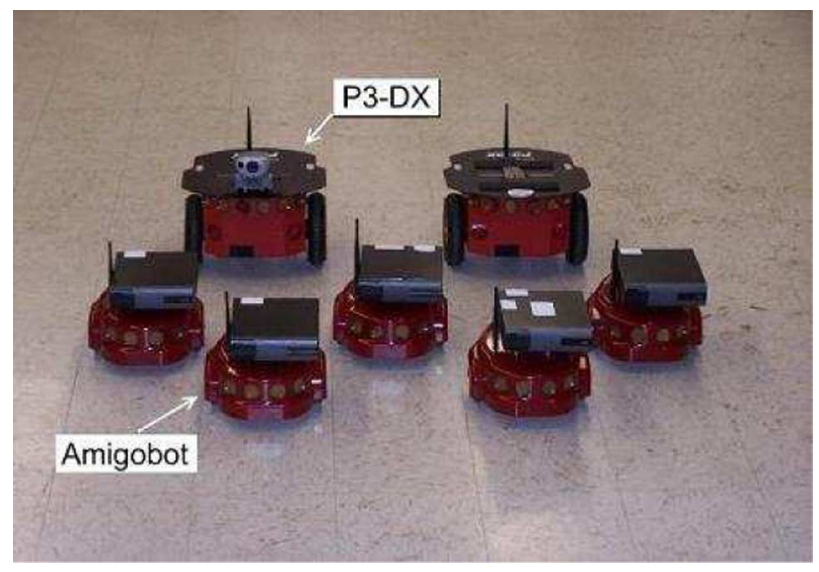

Fig. 6. Multi-vehicle experimental platform at Utah State University.

which can be simplified as $\mu^{2}-\left(1+(1) /\left(\lambda_{i}\right)\right) \mu+(1) /\left(\lambda_{i}\right)-$ $\left(\gamma^{2}\right) /\left(\lambda_{i}^{2}\right)=0$. Because $P$ is symmetric, the eigenvalues of $P$ are real. Therefore, the roots of (15) are all positive if and only if $1+(1) /\left(\lambda_{i}\right)>0$ and $(1) /\left(\lambda_{i}\right)-\left(\gamma^{2}\right) /\left(\lambda_{i}^{2}\right)>0$. After some simplification, we can get that $\gamma<\sqrt{\lambda_{i}}$. Therefore, $P$ is symmetric positive definite if $\gamma<\sqrt{\lambda_{\min }(M)}$.

Similarly, it can be shown that $Q$ is symmetric positive definite if $\gamma<\left(4 \alpha \lambda_{\min }(M)\right) /\left(4+\alpha^{2} \lambda_{\min }(M)\right)$.

Theorem 4.4: Assume that the fixed graph has a united directed spanning tree and the communication patterns among the followers are undirected. Using (11) for (1), if $\alpha$ and $\gamma$ satisfy the conditions in Lemma 4.1 and $\beta>\left\|M^{-1} \Psi_{F}\right\|_{1}$, all followers will converge to the convex hull formed by the leaders, where $\Psi_{F}$ is defined right before (14) and $M$ is defined right after (14).

Proof: Consider the Lyapunov function candidate

$$
\begin{aligned}
V & =\frac{1}{2}\left[\begin{array}{cc}
\tilde{X}_{F}^{T} & \dot{\tilde{X}}_{F}^{T}
\end{array}\right] P\left[\begin{array}{c}
\tilde{X}_{F} \\
\dot{\tilde{X}}_{F}
\end{array}\right] \\
& =\frac{1}{2} \tilde{X}_{F}^{T} \tilde{X}_{F}+\gamma \tilde{X}_{F}^{T} M^{-1} \dot{\tilde{X}}_{F}+\frac{1}{2} \dot{\tilde{X}}_{F}^{T} M^{-1} \dot{\tilde{X}}_{F}
\end{aligned}
$$




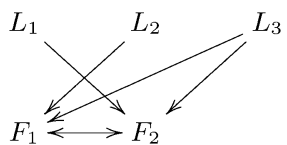

Fig. 7. Network topology for five mobile robots. $L_{i}, i=1, \ldots, 3$, denote the leaders. $F_{i}, i=1, \ldots, 2$, denote the followers.

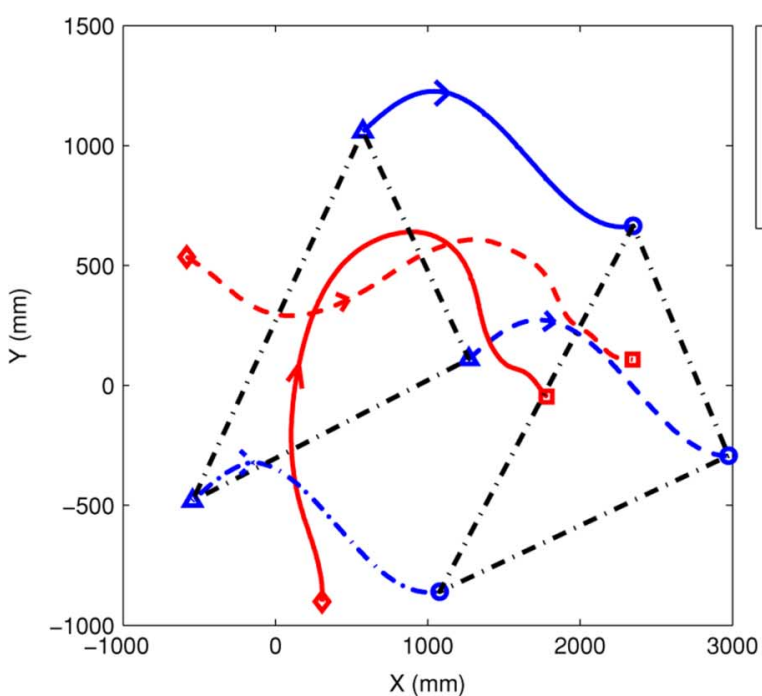

(a)

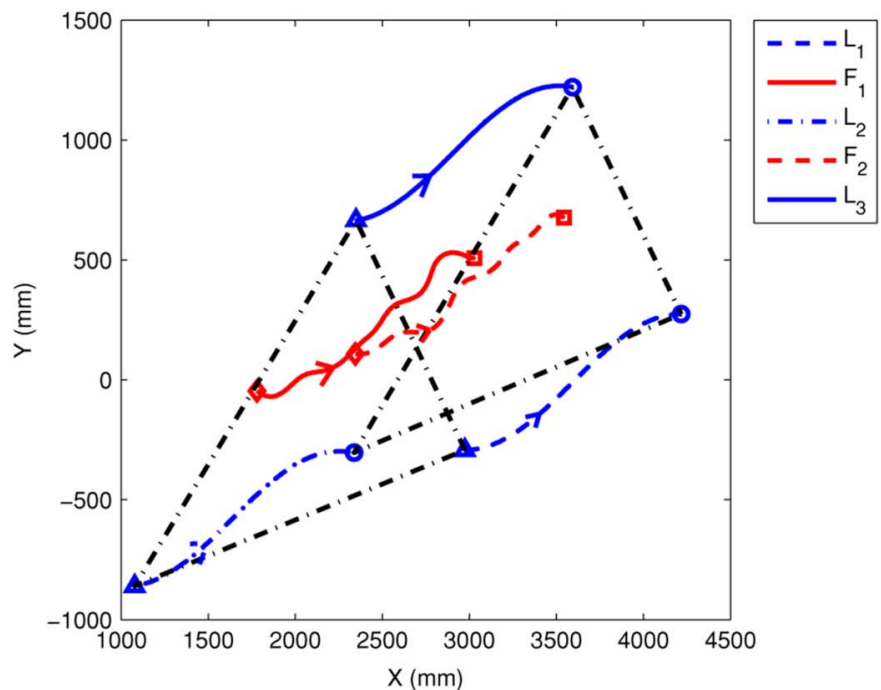

(b)

Fig. 8. Trajectories of the five mobile robots using (5). (a) Part I of the trajectories ( 0 to $25 \mathrm{~s}$ ). (b) Part II of the trajectories ( 25 to $50 \mathrm{~s}$ ).

Note that according to Lemma 4.1, $P$ is symmetric positive definite when $\alpha$ and $\gamma$ satisfy the conditions in Lemma 4.1. Taking derivative of $V$ gives that

$$
\begin{aligned}
\dot{V}= & \tilde{X}_{F}^{T} \dot{\tilde{X}}_{F}+\dot{\tilde{X}}_{F}^{T} M^{-1} \ddot{\tilde{X}}_{F} \\
& +\gamma \dot{\tilde{X}}_{F}^{T} M^{-1} \dot{\tilde{X}}_{F}+\gamma \tilde{X}_{F}^{T} M^{-1} \ddot{\tilde{X}}_{F} \\
= & -\left[\begin{array}{cc}
\tilde{X}_{F}^{T} & \dot{\tilde{X}}_{F}^{T}
\end{array}\right] Q\left[\begin{array}{c}
\tilde{X}_{F} \\
\dot{X}_{F}
\end{array}\right] \\
& -\left(\gamma \tilde{X}_{F}^{T}+\dot{\tilde{X}}_{F}^{T}\right)\left\{\beta \operatorname{sgn}\left[\left(\gamma \tilde{X}_{F}+\dot{\tilde{X}}_{F}\right)\right]+M^{-1} \Psi_{F}\right\}
\end{aligned}
$$

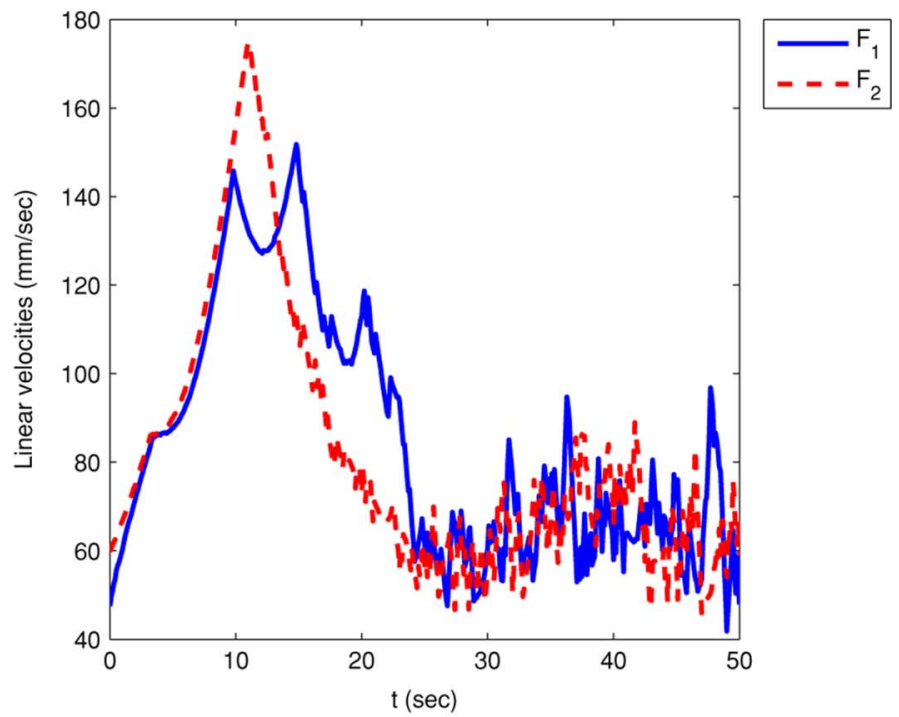

(a)

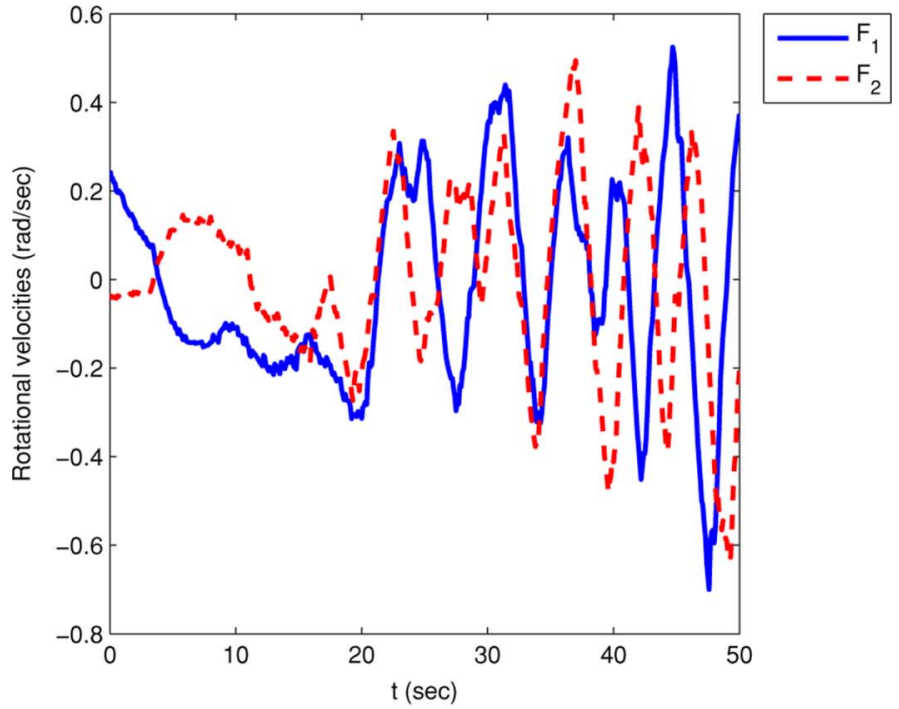

(b)

Fig. 9. Linear and rotational velocities of the two followers using (5). (a) Linear velocities. (b) Rotational velocities.

$$
\begin{aligned}
\leq & -\left[\begin{array}{ll}
\tilde{X}_{F}^{T} & \dot{\tilde{X}}_{F}^{T}
\end{array}\right] Q\left[\begin{array}{l}
\tilde{X}_{F} \\
\tilde{\tilde{X}}_{F}
\end{array}\right] \\
& -\left(\beta-\left\|M^{-1} \Psi_{F}\right\|_{1}\right)\left\|\left(\gamma \tilde{X}_{F}+\dot{\tilde{X}}_{F}\right)\right\|_{1} .
\end{aligned}
$$

Note that according to Lemma 4.1, $Q$ is symmetric positive definite when $\alpha$ and $\gamma$ satisfy conditions in Lemma 4.1. Noting also that $\beta>\left\|M^{-1} \Psi_{F}\right\|_{1}$, it follows that $\dot{V}$ is negative definite. It then follows that $\tilde{X}_{F}=\mathbf{0}$ and $\dot{\tilde{X}}_{F}=\mathbf{0}$. Therefore, $\mathcal{L} X=\mathbf{0}$, which implies that all followers will converge to the convex hull formed by the leaders.

Remark 4.5: Collisions between vehicles may occur when executing the proposed algorithms in Sections III and IV. One approach that might be adopted here is the behavior-based approach where the followers can choose two different actions at each time: collision avoidance and containment control. When two vehicles are quite close, the collision avoidance action can 


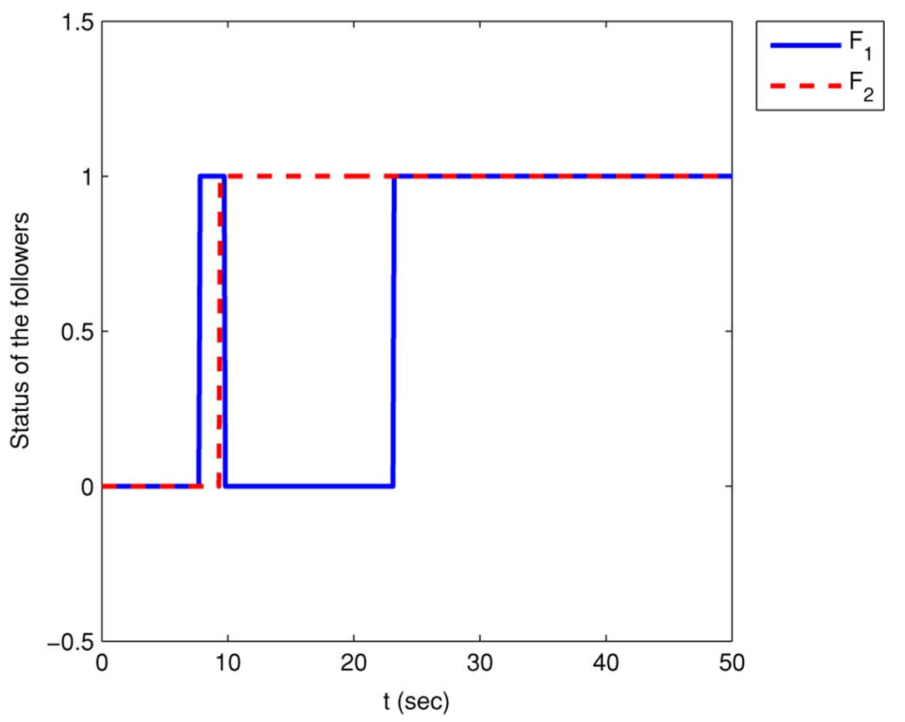

Fig. 10. Status of the two followers. The status is set to 1 when the follower is within the convex hull and 0 otherwise.

be triggered. Otherwise, the containment control action can be triggered. The feasibility of this approach still deserves further investigation. Another approach might be to incorporate the collision avoidance capabilities directly into the control laws by introducing proper potential functions. These will be our future research directions.

\section{Simulation}

In this section, we present several simulation results to validate some theoretical results. We consider a group of vehicles with four leaders and four followers.

When the leaders are stationary, the fixed directed network topology $\mathcal{G}$ is chosen as in Fig. 1(a). It can be noted that $\mathcal{G}$ has a united directed spanning tree. Simulation results using (2) are shown in Fig. 2(a). We can see that all followers will converge to the stationary convex hull formed by the leaders. In the case of switching network topologies, the network topology switches from Fig. 1(b)-(c) every $0.5 \mathrm{~s}$. Note that neither Figs. 1(b) nor (c) has a united directed spanning tree while the union of Figs. 1(b) and (c) has a united directed spanning tree. Simulation results using (2) are shown in Fig. 2(b). We can see that all followers will converge to the stationary convex hull formed by the leaders.

For the algorithms (5) and (7), the fixed network topology is chosen as in Fig. 1(a). Simulation results using (5) are shown in Fig. 3. We can see that all followers will converge to the dynamic convex hull formed by the leaders. Simulation results using (7) are shown in Fig. 4. Note that all followers will converge to the dynamic convex hull formed by the leaders in finite time.

When the velocities of the leaders are nonidentical, the fixed network topology $\mathcal{G}$ is chosen as in Fig. 1(a). Note that $\mathcal{G}$ has a united directed spanning tree and the communication patterns among the followers are undirected. Simulation results using (11) are shown in Fig. 5. It can be noted that all followers will converge to the dynamic convex hull formed by the dynamic leaders.

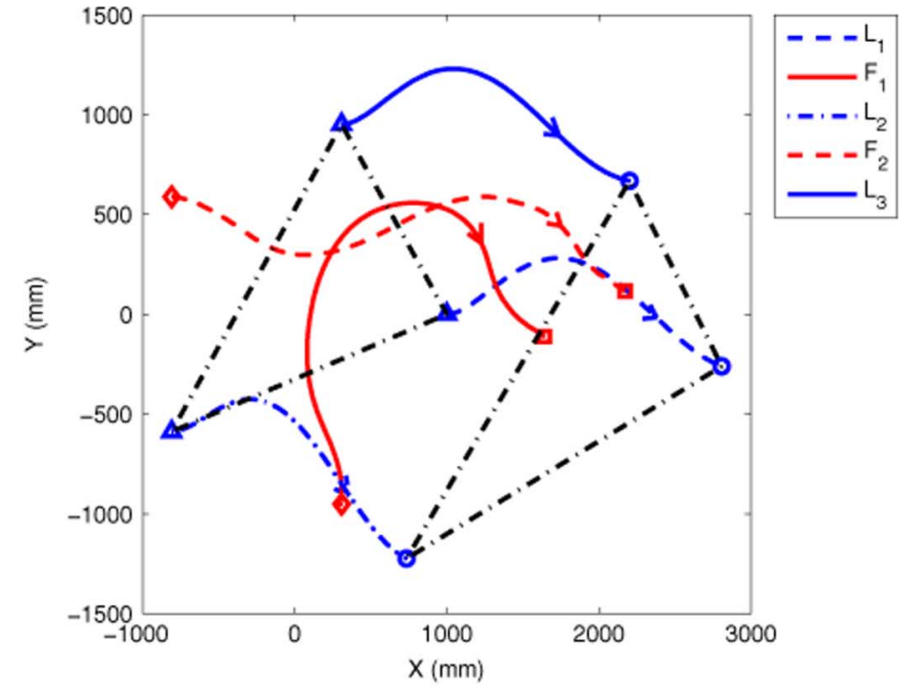

(a)

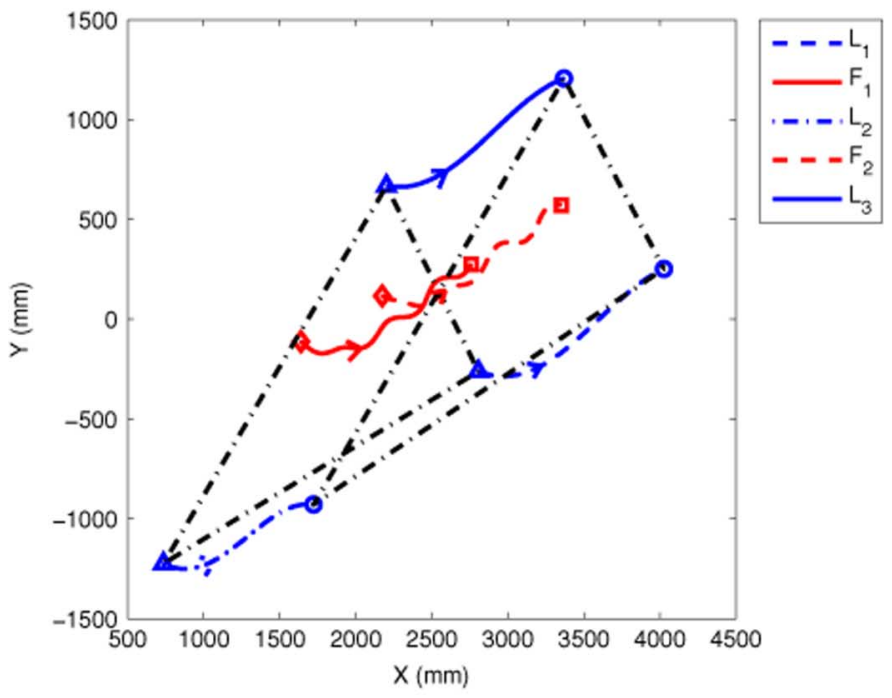

(b)

Fig. 11. Trajectories of the five mobile robots using (11). (a) Part I of the trajectories (0 to $26 \mathrm{~s})$. (b) Part II of the trajectories (26 to $52 \mathrm{~s})$.

\section{EXPERIMENTAL VALIDATION}

In this section, we experimentally validate some of the proposed containment algorithms on a multi-robot platform. In the experiments, five wheeled mobile robots are used to obtain the experimental results. In particular, three robots are designated as the leaders and the other two robots are designated as the followers. We next briefly introduce the experimental platform developed in the Cooperative Vehicle Networks (COVEN) Laboratory at Utah State University.

The textbed in the COVEN Laboratory includes five Amigobots and two P3-DX from the ActivMedia Robotics as shown in Fig. 6. Both the Amigobots and P3-DX are similar in terms of functionalities. Each robot has a differential-drive system with rear caster, high precision wheel encoders, and eight sonar positioned around the robot. The robots can calculate their positions and orientations based on the encoders. The 


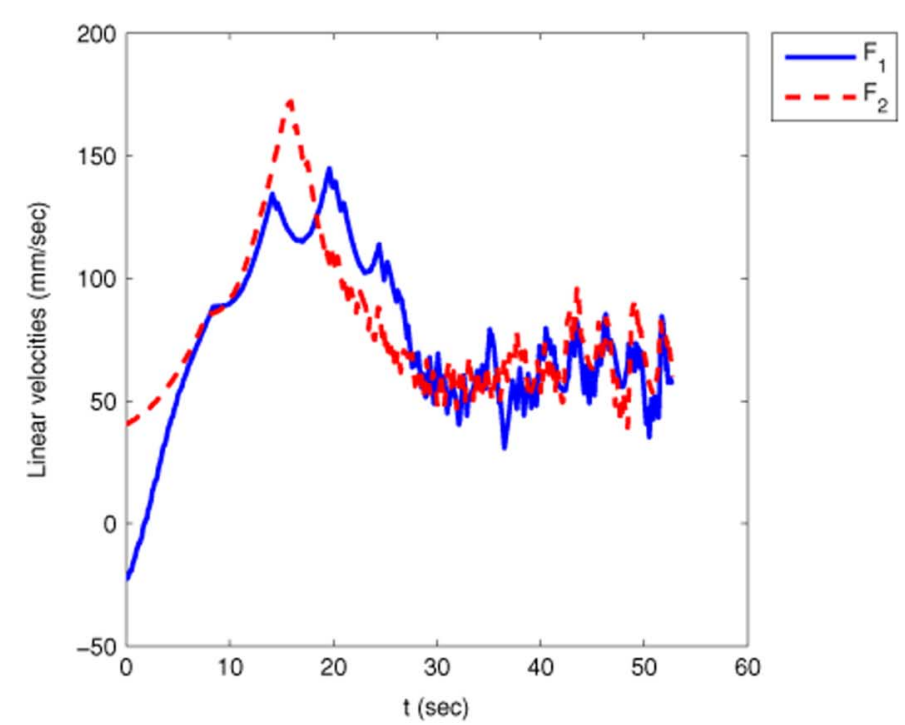

(a)

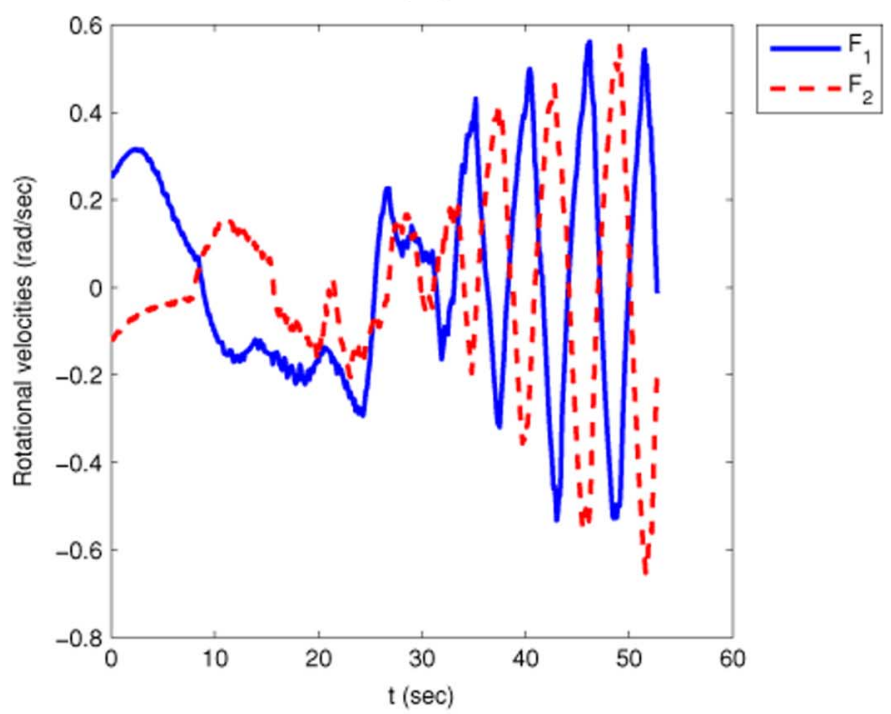

(b)

Fig. 12. Linear and rotational velocities of the two followers using (11). (a) Linear velocities. (c) Rotational velocities.

eight sonar can be used for localization and detection of obstacles. Although the Amigobots have eight sonar, the encoders are more reliable for our experiments; whereas the eight sonar have proven more reliable for environmental navigation. The maximum speed for the AmigoBots is $1 \mathrm{~m} / \mathrm{s}$ and the AmigoBots can climb a $1.5 \%$ incline.

In order to control multiple mobile robots under various network topologies, a control software was developed to emulate a limited or even changing network topology. The control platform can be divided into two layers [26]. The top layer is responsible for network topology setting, control algorithm implementation, and bidirectional communication with the onboard micro-controller. The bottom layer is responsible for sensor data acquisition and direct PID loop control where both linear and rotational velocity commands are generated and executed.

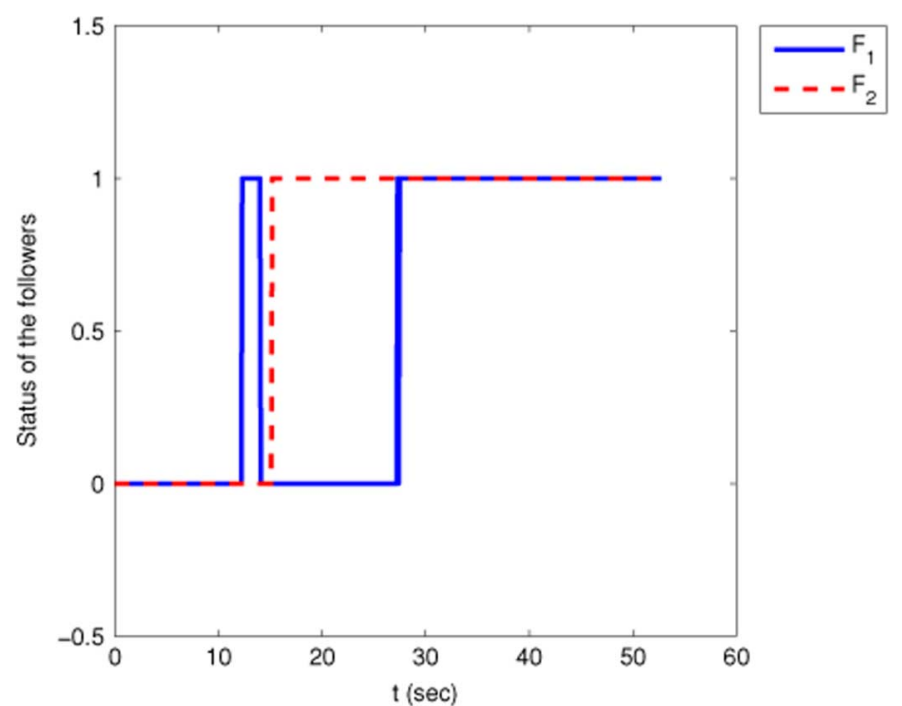

Fig. 13. Status of the two followers. The status is set to 1 when the follower is within the convex hull and 0 otherwise.

The system dynamics of the wheeled mobile robots can be described as

$$
\dot{x}_{i}=v_{i} \cos \left(\theta_{i}\right), \quad \dot{y}_{i}=v_{i} \sin \left(\theta_{i}\right), \quad \dot{\theta}_{i}=\omega_{i}
$$

where $\left(x_{i}, y_{i}\right)$ is the position of the center of the $i$ th robot, $\theta_{i}$ is the orientation of the $i$ th robot, and $v_{i}$ and $w_{i}$ are the linear and rotational velocities of the $i$ th robot. To avoid using the nonlinear dynamics (16), we feedback linearize (16) for a fixed point off the center of the wheel axis denoted as $\left(x_{h i}, y_{h i}\right)$, where $x_{h i}=x_{i}+d_{i} \cos \left(\theta_{i}\right)$ and $y_{h i}=y_{i}+d_{i} \sin \left(\theta_{i}\right)$ with $d=0.15 \mathrm{~m}$. By letting

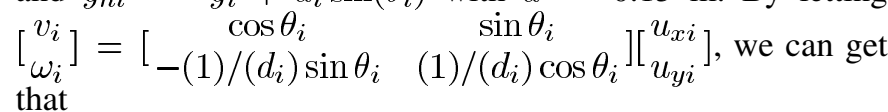

$$
\left[\begin{array}{c}
\dot{x}_{h i} \\
\dot{y}_{h i}
\end{array}\right]=\left[\begin{array}{l}
u_{x i} \\
u_{y i}
\end{array}\right] .
$$

Note that (17) is a single-integrator kinematics model. By letting $\dot{u}_{x i}=\tau_{x i}$ and $\dot{u}_{y i}=\tau_{y i}$, a double-integrator dynamics model can be obtained by designing the control inputs $\tau_{x i}$ and $\tau_{y i}$.

The network topology is chosen as in Fig. 7. It can be noted from Fig. 7 that the network topology has a united directed spanning tree. In the following, we use triangles and circles to denote, respectively, the starting and ending positions of the leaders, and diamonds and squares to denote, respectively, the starting and ending positions of the followers.

When the velocities of leaders are identical, experimental results using (5) are given in Fig. 8 where Fig. 8(a) and (b) together show the trajectories of the five robots. In each subfigure, two snapshots are presented to show, respectively, the starting positions of the five robots as well as the convex hull formed by the leaders and the ending positions of the five robots as well as the convex hull formed by the leaders. The linear and rotational velocities of the two followers are shown in Fig. 9. In order to better demonstrate whether the followers will converge to the convex hull formed by the leaders, Fig. 10 shows the status of the two followers using (5). It can be seen from Fig. 10 that the 
two followers moved to the convex hull formed by the leaders, which is consistent with the results in Theorem 4.1.

When the velocities of leaders are nonidentical, experimental results using (11) are given in Fig. 11, where Fig. 11(a) and (b) together show the trajectories of the five robots. In each subfigure, we also use two snapshots to show, respectively, the starting positions of the five robots as well as the convex hull formed by the leaders and the ending positions of the five robots as well as the convex hull formed by the leaders. The linear and rotational velocities of the two followers are shown in Fig. 12. Similar to the identical velocity case, we use Fig. 13 to show the status of the two followers using (11). It can be seen from Fig. 13 that the two followers moved to the convex hull formed by the leaders, which is consistent with the results in Theorem 4.4.

\section{CONCLUSION}

In this brief, we studied distributed containment control for double-integrator dynamics in the presence of both stationary and dynamic leaders. First, we proposed a distributed containment control algorithm in the presence of multiple stationary leaders and presented conditions on the network topology and the control gains to guarantee asymptotic containment control. We then proposed two distributed containment control algorithms in the presence of multiple dynamic leaders with an identical velocity to solve, respectively, asymptotic containment control and finite-time containment control. When the dynamic leaders have nonidentical velocities, we proposed a distributed containment control algorithm and derived sufficient conditions on the network topology and the control gains to guarantee asymptotic containment control. Both simulation results and experimental results on a multi-robot platform were provided to show the effectiveness of some theoretical results. The experimental movies of this brief can be found at http://www.neng.usu.edu/ece/faculty/wren/videos/Amigobots/containment-same-vel.MOV and http://www.neng.usu.edu/ece/faculty/wren/videos/Amigobots/ containment-vary-vel.MOV.

\section{REFERENCES}

[1] A. Jadbabaie, J. Lin, and A. S. Morse, "Coordination of groups of mobile autonomous agents using nearest neighbor rules," IEEE Trans. Autom. Control, vol. 48, no. 6, pp. 988-1001, Jun. 2003.

[2] F. Xiao and L. Wang, "Asynchronous consensus in continuous-time multi-agent systems with switching topology and time-varying delays," IEEE Trans. Autom. Control, vol. 53, no. 8, pp. 1804-1816, Aug. 2008.

[3] M. Cao, A. S. Morse, and B. D. O. Anderson, "Agreeing asynchronously," IEEE Trans. Autom. Control, vol. 53, no. 8, pp. 1826-1838, Aug. 2008.
[4] G. Lafferriere, A. Williams, J. Caughman, and J. J. P. Veerman, "Decentralized control of vehicle formations," Syst. Control Lett., vol. 54, no. 9, pp. 899-910, 2005.

[5] R. S. Smith and F. Y. Hadaegh, "Closed-loop dynamics of cooperative vehicle formations with parallel estimators and communication," IEEE Trans. Autom. Control, vol. 52, no. 8, pp. 1404-1414, Aug. 2007.

[6] R. Sepulchre, D. A. Paley, and N. E. Leonard, "Stabilization of planar collective motion with limited communication," IEEE Trans. Autom. Control, vol. 53, no. 3, pp. 706-719, Mar. 2008.

[7] J. Cortes, S. Martinez, and F. Bullo, "Robust rendezvous for mobile autonomous agents via proximity graphs in arbitrary dimensions," IEEE Trans. Autom. Control, vol. 51, no. 8, pp. 1289-1298, Aug. 2006.

[8] D. V. Dimarogonas and K. J. Kyriakopoulos, "On the rendezvous problem for multiple nonholonomic agents," IEEE Trans. Autom. Control, vol. 52, no. 5, pp. 916-922, May 2007.

[9] R. Olfati-Saber, "Flocking for multi-agent dynamic systems: Algorithms and theory," IEEE Trans. Autom. Control, vol. 51, no. 3, pp. 401-420, Mar. 2006.

[10] H. G. Tanner, A. Jadbabaie, and G. J. Pappas, "Flocking in fixed and switching networks," IEEE Trans. Autom. Control, vol. 52, no. 5, pp. 863-868, May 2007.

[11] H. Su, X. Wang, and Z. Lin, "Flocking of multi-agents with a virtual leader," IEEE Trans. Autom. Control, vol. 54, no. 2, pp. 293-307, Feb. 2009.

[12] R. Olfati-Saber, J. A. Fax, and R. M. Murray, "Consensus and cooperation in networked multi-agent systems," Proc. IEEE, vol. 95, no. 1, pp. 215-233, Jan. 2007.

[13] W. Ren, R. W. Beard, and E. M. Atkins, "Information consensus in multivehicle cooperative control," IEEE Control Syst. Mag., vol. 27, no. 2, pp. 71-82, Feb. 2007.

[14] Y. Hong, J. Hu, and L. Gao, "Tracking control for multi-agent consensus with an active leader and variable topology," Automatica, vol. 42, no. 7, pp. 1177-1182, 2006.

[15] Y. Hong, G. Chen, and L. Bushnell, "Distributed observers design for leader-following control of multi-agent networks," Automatica, vol. 44, no. 3, pp. 846-850, 2008.

[16] W. Ren, "Multi-vehicle consensus with a time-varying reference state," Syst. Control Lett., vol. 56, no. 7-8, pp. 474-483, 2007.

[17] Y. Cao, W. Ren, and Y. Li, "Distributed discrete-time coordinated tracking with a time-varying reference state and limited communication," Automatica, vol. 45, no. 5, pp. 1299-1305, 2009.

[18] K. Peng and Y. Yang, "Leader-following consensus problem with a varying-velocity leader and time-varying delays," Phys. A, vol. 388, no. 2-3, pp. 193-208, 2009.

[19] M. Ji, G. Ferrari-Trecate, M. Egerstedt, and A. Buffa, "Containment control in mobile networks," IEEE Trans. Autom. Control, vol. 53, no. 8, pp. 1972-1975, Aug. 2008.

[20] Y. Cao and W. Ren, "Containment control with multiple stationary or dynamic leaders under a directed interaction graph," in Proc. IEEE Conf. Decision Control, Shanghai, China, Dec. 2009, pp. 3014-3019.

[21] Y. Cao, D. Stuart, W. Ren, and Z. Meng, "Distributed containment control for double-integrator dynamics: Algorithms and experiments," in Proc. Amer. Control Conf., Jun./Jul. 2010, pp. 3830-3835.

[22] L. Moreau, "Stability of multi-agent systems with time-dependent communication links," IEEE Trans. Autom. Control, vol. 50, no. 2, pp. 169-182, Feb. 2005.

[23] J. P. Aubin and A. Cellina, Differential Inclusions: Set-Valued Maps and Viability Theory. New York: Springer, 1984.

[24] A. F. Filippov, Differential Equations With Discontinuous Righthand Sides. Norwell, MA: Kluwer, 1988.

[25] S. P. Bhat and D. S. Bernstein, "Continuous finite-time stabilization of the translational and rotational double integrators," IEEE Trans. Autom. Control, vol. 43, no. 5, pp. 678-682, May 1998.

[26] Y. Cao, W. Ren, N. Sorensen, L. Ballard, A. Reiter, and J. Kennedy, "Experiments in consensus-based distributed cooperative control of multiple mobile robots," in Proc. IEEE Conf. Mechatron. Autom., Harbin, China, Aug. 2007, pp. 2819-2824. 